\title{
FACTORS AFFECTING OUTCOME OF RCTS OF KETAMINE FOR POSTOPERATIVE PAIN
}

William PS McKay MD, Dept of Anesthesia, University of Saskatchewan

RUH, 103 Hospital Drive, Saskatoon SK, S7N 0W8

INTRODUCTION Outcomes of randomised controlled trials (RCTs) of adjunctive lowdose ketamine for postoperative pain control are surprisingly disparate. An unexpected negative study at our institution prompted a re-examination of other studies. Tabulation of factors reported in RCTs of a quality to be included in systematic reviews $[1,2,3]$ suggests reasons for the disparity.

METHODS RCTs, included in the systematic reviews, that involved intravenous ketamine and morphine (excluding epidural analgesia), were examined for side effects and efficacy. Factors tabulated for efficacy, defined as lower pain scores and/or decreased use of morphine: P-value (where possible, true P-values were calculated); ketamine dose; induction narcotic dose; non-steroidal anti-inflammatory drug (NSAID) use; timing of administration; type/site of surgery; painfulness of surgery; efficacy in the first four hours after surgery; and prolonged efficacy. Factors tabulated for side effects: ketamine dose, preoperative sedative used, and postoperative hallucinations or delirium.

RESULTS 1) P-values fell between 0.01 and 0.1 in only three of 22 studies. The rest were an order of magnitude or more greater or smaller. 2) Ketamine dose, narcotics, and NSAIDS: a single injection less than $75 \mu / \mathrm{kg}$ of ketamine was not efficacious. Otherwise, neither ketamine dose, nor induction narcotic dose, nor NSAIDS was related to ketamine efficacy. 3) Timing of administration: in all 12 studies where ketamine was given after surgical pain, it was efficacious. In five studies, the effect lasted more than 4 hours; in 3 studies, 48 hours. 4) Type of surgery: nine of ten studies with surgery involving somatic (as opposed to visceral) pain had a positive outcome. Upper abdominal surgery showed more benefit than lower. 5) Painfulness of surgery: three out of four studies report efficacy for a severe-pain probability $\geq 0.5$.

DISCUSSION 1) The distribution of P-values suggests different experimental effects in negative and positive studies. 2) Timing of administration: far from being pre-emptive of pain, ketamine worked best when given after a painful stimulus. 3) Somatic surgical pain is better attenuated by ketamine than is visceral pain; 4) more painful surgery better than less painful. 5) Single injections work as well as infusions. 6) Benzodiazepines prevented hallucinations and delirium.

REFERENCES [1] Anesth Analg 2004; 99: 482 - 95. [2] Anesth Analg 2004; 98: 1385 1400. [3] Anesthesiology 2002; 96: 725 -741. 